\title{
Rationality of using whole-body computed tomography in trauma patients
}

\author{
Wojciech Wierzchołowski ${ }^{1 A, B, C, D, E, F}$, Jerzy Michał Walecki ${ }^{2 A, D, E, F}$, Tomasz Latos ${ }^{2 A, D, E, F}$ \\ 'Department of Radiology, Medical College, University of Zielona Góra, Zielona Góra, Poland \\ ${ }^{2}$ Department of Radiology, Medical Centre for Postgraduate Education, Warsaw, Poland
}

\section{Abstract}

Purpose: Identification of the group of patients meeting the criteria of a severe multiple trauma (polytrauma) among those admitted to the regional trauma centre and subjected to whole-body computed tomography (WBCT) due to injuries. Identification the patients for whom WBCT was the optimal choice.

Material and methods: Retrospective analysis of the data of 303 patients undergoing WBCT in the period 2015-2017 with assessment of the injury severity based on the abbreviated injury scale (AIS) and injury severity score (ISS).

Results: Among 303 patients undergoing WBCT due to trauma, multiple injuries with ISS $>15$ were found in 74 individuals (24.4\%). After excluding patients whose limb fractures increased ISS above 15, the group decreased to 63 patients $(20.8 \%)$. For these patients, the WBCT may be described as the optimal imaging method at an early stage of management.

Conclusions: Trauma patients are too often qualified for WBCT. Exposure to a high dose of ionising radiation associated with this modality requires critical analysis of the scope of indications and rules of conduct for trauma teams.

Key words: trauma, whole-body computed tomography (WBCT), polytrauma, injury severity score (ISS).

\section{Introduction}

The implementation of whole-body computed tomography (WBCT) in the sequence of diagnostic procedures has significantly changed the management of trauma patients. Compared to the standard diagnostic algorithm defined by the principles of advanced trauma life support (ATLS), which is based on X-ray images, extended focused assessment with sonography for trauma (eFAST), and computed tomography of the head, WBCT allows the visualisation of injuries in different areas of the body much more quickly and with greater accuracy. This significantly facilitates establishment of the optimal therapeutic strategy [1].

There are no standardised and universally approved criteria for qualifying patients for WBCT in trauma cen- tres. They may vary not only between countries but even between individual centres [2]. In general, these criteria are based on an assessment of the vital signs of the patient and the mechanism of trauma suggesting its high-energy nature, and they sometimes also include an element of clinical judgement raising the suspicion of a serious injury. There are also differences in terms of the limits of vital signs justifying referral of a trauma patient for WBCT. The same applies to the characteristics of the circumstances indicating the likelihood of a high-energy nature of the injury $[3,4]$.

In each case, however, the qualification criteria are intended to serve a single purpose: to identify as precisely as possible those patients for whom WBCT is the modality of choice in cases of severe injuries in different areas of the body, referred to as polytrauma. It should be stressed that the introduction of this concept into clinical language

Correspondence address:

Dr. Wojciech Wierzchołowski, Department of Radiology, Medical College, University of Zielona Góra, 26Zyty Str., 65-046 Zielona Góra, Poland, e-mail: wojtwie@wp.pl

Authors' contribution:

A Study design · B Data collection · C Statistical analysis · D Data interpretation · E Manuscript preparation · F Literature search · G Funds collection 
and its significance stems from the observation that the expected mortality in patients diagnosed with polytrauma is higher than a simple summation of probable mortality rates associated with individual injuries [5].

For some time now, we have been observing an increasingly liberal attitude towards indications for WBCT performed in trauma patients. This trend results in a visible increase in the number of scans showing either no traumatic lesions or injuries limited to one region of the body, which are sometimes clinically insignificant [6,7]. This has serious consequences both for patients, due to the potential consequences of exposure to a high dose of ionising radiation, and for the entire healthcare system, due to the high costs of WBCT scanning [8]. On the other hand, proponents of a less restrictive approach to the use of this test in trauma patients point to the key role of WBCT in imagining serious injuries in those patients whose clinical condition is initially good $[9,10]$. Without doubt, the significance of WBCT as a primary diagnostic tool in the early phase of managing trauma patients was also established by studies proving its positive impact on reducing mortality [11-13]. However, this problem is still being debated. Some recent publications have called into question the advantage of WBCT over the standard model of radiological diagnostic procedure recommended in the ATLS protocol in terms of its significance for reducing mortality [14].

Problems related to these issues are of particular importance in trauma centres, which carry out the greatest number of WBCT examinations. In Poland, there are currently 15 regional trauma centres, one of which is the University Hospital in Zielona Gora. The operations of the centre cover an area of about $14,000 \mathrm{~km}^{2}$ with a population of about one million people.

The purpose of our study was twofold: first, to determine the actual size of the group of patients meeting the criteria of severe multiple injuries (polytrauma) and subjected to WBCT due to a suspicion of injuries; and second, to attempt to identify the group for which this modality was indeed the optimal choice.

\section{Material and methods}

The study was retrospective. An assessment of WBCT scans performed on trauma patients in the period from July 2015 to June 2017 was made. Patients were referred for WBCT from the Emergency Department of the University Hospital in Zielona Gora. In the discussed period, 315 examinations were performed, of which 303 were included for further analysis. The reasons for exclusion of 12 scans were incomplete data (8) or performance of scans not immediately after hospital admission, also in patients transferred from other hospitals (4).

All scans were performed on a 64-row computed tomography (CT) scanner (General Electric Lightspeed) in the Department of Diagnostic Imaging according to the uniform protocol presented in Table 1 .
Data were collected on the patient demographics, hospitalisation time, and the circumstances of the injury. In each patient, the body location of traumatic lesions was determined (head and neck, chest, abdominal cavity, and pelvis), including changes in individual sections of the spine for the corresponding areas.

As in the case of many other trauma centres, the group of patients meeting polytrauma criteria was established using the abbreviated injury scale (AIS 2005, update 2008), followed by retrospective calculation of the injury severity score (ISS). The ISS for each patient was calculated based on imaging procedure results and medical records, including protocols of surgical procedures.

The literature provides various definitions of the term polytrauma, based on different abbreviated injury scale (AIS)/ISS values for injuries occurring in at least two body regions. In clinical trials, the most common cut-off values are ISS $>15$ and ISS $>17$, while some authors suggest adopting the criterion of AIS $>2$ for injuries in at least two body regions [15]. This study adopted the lowest cutoff value, i.e. ISS $>15$.

To establish the actual size of the group of patients for whom WBCT was the best choice in terms of meeting polytrauma criteria, the group of all patients with multiple injuries with ISS $>15$ was further reduced by excluding patients whose limb injuries resulted in ISS $>15$. Limb fractures were diagnosed by using an X-ray, and WBCT was not used to refine the diagnosis of the nature of fractures or vascular injury.

\section{Results}

Of the 303 patients, there were 231 men and 72 women; the average age was 39.5 years. A group of 23 patients $(7.6 \%)$ were children up to 15 years of age. There were 193 patients who were victims of road traffic accidents, including 36 pedestrians. Another 81 patients were victims of falls. Finally, 29 patients were classified into the group 'Other events', which included victims of violent acts and individuals found with symptoms of injuries sustained in unknown circumstances.

Overall, 247 patients ( $81.5 \%$ ) were hospitalised, of whom 68 patients $(22.4 \%)$ were admitted to the Intensive Care Unit. Thirty-three patients died (10.9\%), while 56 patients (18.5\%) were discharged from hospital immediately after being examined and receiving the necessary medical assistance. The data characterising the studied group of patients are shown in Table 2.

Traumatic lesions were diagnosed in 221 patients (72.9\%), but only in 114 patients (37.6\%) were injuries found in at least two body regions. No injury was reported in 82 patients $(27.1 \%)$ (Figure 1$)$.

The mean ISS for the entire group of patients was 12.08 , and a median of 9.74 patients $(24.4 \%)$ had injuries in at least two body regions with ISS $>15$. Exclusion of patients with limb fractures increasing ISS $>15$ reduced 
Table 1. Whole-body computed tomography protocol in trauma examination

\begin{tabular}{|c|c|c|c|c|}
\hline Body area & Slice thickness & Reformats & \multicolumn{2}{|c|}{ Contrast medium* } \\
\hline Head & $1.25 \mathrm{~mm}$ & & & \\
\hline Neck & $1.25 \mathrm{~mm}$ & Coronal and sagittal $3.75 \mathrm{Mm}$ & \multirow{4}{*}{$\begin{array}{l}\text { Arterial phase } 25-30 \mathrm{~s} \\
\text { after administration } \\
\text { of contrast medium }\end{array}$} & \\
\hline Chest & $1.25 \mathrm{~mm}$ & Coronal and sagittal $3.75 \mathrm{Mm}$ & & \\
\hline Abdominal cavity & $1.25 \mathrm{~mm}$ & Coronal and sagittal $3.75 \mathrm{Mm}$ & & \multirow{2}{*}{$\begin{array}{l}\text { Venous phase } 55-60 \mathrm{~s} \\
\text { after administration of contrast medium }\end{array}$} \\
\hline Pelvis & $1.25 \mathrm{~mm}$ & Coronal and sagittal $3.75 \mathrm{Mm}$ & & \\
\hline
\end{tabular}

Lower limit of the study range: proximal one-third of femoral shafts. *Administration of the contrast medium: $100 \mathrm{ml}, 3-4 \mathrm{ml} / \mathrm{s}$ using an automatic injector.

Table 2. Patient data

\begin{tabular}{|l|c|c|c|c|}
\hline Parameter & Total & Victims of traffic accidents & Victims of falls & Other \\
\hline Number of patients & 303 & 193 & 81 & 29 \\
\hline Men, $n(\%)$ & $231(76.2)$ & $143(74.1)$ & $64(79.0)$ & $24(82.7)$ \\
\hline Women, $n$ (\%) & $72(23.8)$ & $50(25.9)$ & $17(21.0)$ & $5(17.3)$ \\
\hline Average age, range (years) & $39.5,2-94$ & $35.5,2-92$ & $47.7,2-94$ & $44.3,18-82$ \\
\hline Hospitalised, $n$ (\%) & $247(81.5)$ & $160(82.9)$ & $67(82.7)$ & $20(69.0)$ \\
\hline Admitted to ICU & $68(22.4)$ & $49(25.4)$ & $10(12.3)$ & $9(31.0)$ \\
\hline Deaths, $n(\%)$ & $33(10.9)$ & $20(10.4)$ & $8(9.9)$ & $5(17.2)$ \\
\hline
\end{tabular}

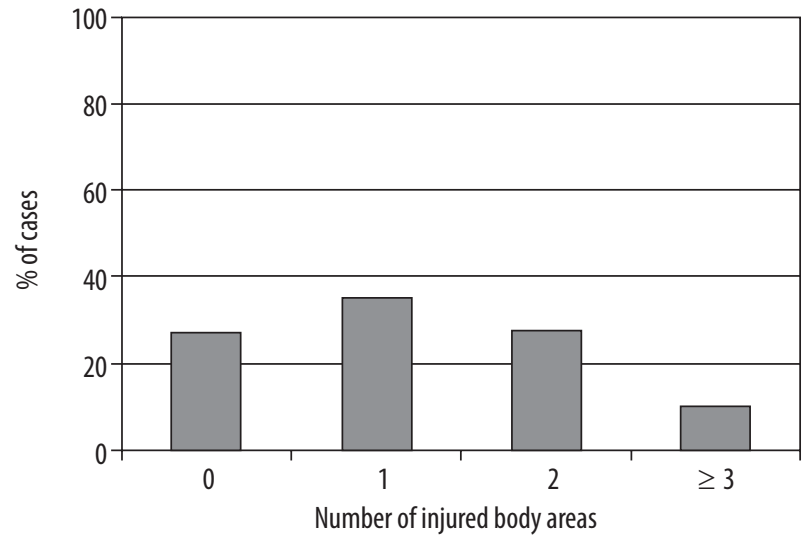

Figure 1. Body areas with traumatic lesions

Table 3. Values of injury severity score (ISS)

\begin{tabular}{|l|c|c|c|c|}
\hline Parameter & $\begin{array}{c}\text { Number } \\
\text { of patients }\end{array}$ & $\begin{array}{c}\text { Average } \\
\text { ISS }( \pm \text { SD) }\end{array}$ & $\begin{array}{c}\text { Median } \\
\text { ISS }\end{array}$ & $\begin{array}{c}\text { ISS } \\
\text { range }\end{array}$ \\
\hline The whole group & 303 & $12.08( \pm 10.86)$ & 9 & $0-50$ \\
\hline $\begin{array}{l}\text { Patients with } \\
\text { ISS }>15\end{array}$ & $74(24.4 \%)$ & $25.78( \pm 9.41)$ & 22 & $0-50$ \\
\hline $\begin{array}{l}\text { Patients with } \\
\text { ISS }>15 \text { after } \\
\text { excluding patients } \\
\text { with limb fractures }\end{array}$ & $63(20.8 \%)$ & $27.03( \pm 9.64)$ & 24 & $0-50$ \\
\hline
\end{tabular}

this group to 63 patients (20.8\%). Detailed data are shown in Tables 3 and 4.

The chest was the most frequently injured body region with AIS $>2$ in the group of polytrauma patients (Table 5).
Table 4. List of patients with extremity injuries raising injury severity score (ISS) over 15

\begin{tabular}{|c|c|c|c|}
\hline Patients & $\begin{array}{l}\text { Original ISS } \\
\text { including AIS } \\
\text { score of limb } \\
\text { fractures }\end{array}$ & $\begin{array}{l}\text { Type of fracture, } \\
\text { AIS score }\end{array}$ & $\begin{array}{l}\text { Recalculated } \\
\text { ISS }\end{array}$ \\
\hline DS & 18 & $\begin{array}{c}\text { Open tibia fracture } \\
3\end{array}$ & 9 \\
\hline SC & 17 & $\begin{array}{c}\text { Open radius fracture } \\
3\end{array}$ & 8 \\
\hline$K D$ & 22 & $\begin{array}{c}\text { Femur fracture } \\
3\end{array}$ & 13 \\
\hline JT & 18 & $\begin{array}{c}\text { Femur fracture } \\
3\end{array}$ & 9 \\
\hline GG & 17 & $\begin{array}{c}\text { Femur fracture } \\
3\end{array}$ & 8 \\
\hline $\mathrm{RL}$ & 18 & $\begin{array}{c}\text { Femur fracture } \\
3\end{array}$ & 9 \\
\hline $\mathrm{DH}$ & 17 & $\begin{array}{c}\text { Femur fracture } \\
3\end{array}$ & 8 \\
\hline SK & 17 & $\begin{array}{c}\text { Tibia fracture } \\
2\end{array}$ & 13 \\
\hline WJ & 22 & $\begin{array}{c}\text { Femur fracture } \\
3\end{array}$ & 13 \\
\hline WG & 22 & $\begin{array}{c}\text { Femur fracture } \\
3\end{array}$ & 13 \\
\hline$A B$ & 17 & $\begin{array}{c}\text { Humerus fracture } \\
2\end{array}$ & 13 \\
\hline
\end{tabular}


Table 5. Occurrence of abbreviated injury scale (AIS) $>2$ injuries in different body regions of polytrauma patients

\begin{tabular}{|l|c|}
\hline Body regions & Polytrauma patients \\
\hline Head and neck injuries with AIS > & $38 / 74(51.3 \%)$ \\
\hline Chest injuries with AIS > 2 & $49 / 74(63.5 \%)$ \\
\hline Abdominal cavity injuries with AIS > 2 & $26 / 74(35.1 \%)$ \\
\hline Pelvis and limb injuries with AIS > 2 & $24 / 74(32.4 \%)$ \\
\hline
\end{tabular}

\section{Discussion}

The integration of WBCT into the early diagnostic protocol was a turning point, changing the pattern of initial trauma management. In daily practice, WBCT has become an integral part of primary survey as defined by the rules of the ATLS protocol, eliminating in many cases the need to carry out a secondary survey [16]. Such a procedure, in principle not formally included in the rules of trauma protocols, resulted from daily observation, and was additionally confirmed in scientific studies, indicating that the use of WBCT significantly reduces the time from patient's arrival to the emergency department until the diagnosis is made, thus allowing the treatment plan to be outlined and its priorities set out much faster [1]. This is crucial for patients with multiple severe injuries, for whom time is a critical factor.

The formulation of structured criteria for qualification of trauma patients for WBCT, based on a clinical examination and evaluation of vital signs, and considering the mechanism and circumstances of the injury, was intended to identify patients who probably suffered serious injuries and for whom such scanning would be the optimal imaging modality. In practice, however, as shonw by literature data, the percentages of patients undergoing WBCT for a trauma with no reported injuries are high and fall in the range from $28 \%$ to as much as $42 \%[17,18]$. In the case of our centre, this value was about $27 \%$ and was very similar to the figure presented in a study by Błaż et al. [17].

Analysis of the severity of injuries using AIS and ISS brings additional valuable information because it allows us to determine the number of patients for whom WBCT was actually the best choice, considering their exposure to a high dose of ionising radiation and, what is significant in the broader economic context, the high cost of this examination.

Without doubt, the percentage of patients whose injuries meet the definition of polytrauma specified as ISS $>15$ is low in this analysis, at less than $25 \%$.

In our opinion, the issue of excessively broad treatment of indications should be looked at with great cau- tion and from the right perspective considering the understandable concerns of trauma team doctors about legal consequences.

It should be emphasised that radiologists are also responsible for patients when it comes to choosing diagnostic modalities. Obviously, they know best the potential of the respective methods, but also, out of the entire trauma team, they understand best the meaning of the "as low as reasonably achievable" (ALARA) principle and the resulting need to strive to minimise patient exposure to ionising radiation. Hence the belief that radiologists must be well acquainted with the indications for WBCT and should become full members of trauma teams. We think that this would allow their more active participation in the decision-making process, giving them real influence on the selection of the optimal diagnostic method(s) in a particular clinical situation.

Our study indicates that in terms of the extent and severity of the recognised injuries, WBCT is a tool that is used far too often.

The presented results suggest the need for a deeper analysis of the indications for WBCT in trauma patients in the daily work of trauma centres. They also point to the need for a critical look at the extent to which the current criteria allow selection of patients for whom this diagnostic method is in fact the optimal choice.

A limitation of the study is its single-centre design. In our opinion, it would be worthwhile to try to create a much larger database as part of the cooperation between regional trauma centres, to estimate the real scale of the problem and to take actions aimed at rational use of WBCT in diagnosis of traumatic lesions.

\section{Conclusions}

In the evaluated material, only $24.4 \%$ of trauma patients subjected to WBCT met the polytrauma criteria specified by ISS $>15$ and the presence of injuries in at least two body regions.

In patients with polytrauma diagnosis, injuries with AIS $>2$ occurred most often in the chest area and were least frequent in the pelvis and limb areas.

In terms of the extent and severity of injuries, WBCT can be considered the optimal choice only for $20.8 \%$ of patients from the entire group.

\section{Conflict of interest}

The authors report no conflict of interest. 


\section{References}

1. Wurmb TE, Frühwald P, Hopfner W, et al. Whole-body multislice computed tomography as the first line diagnostic tool in patients with multiple injuries: the focus on time. J Trauma 2009; 66: 658-665.

2. Treskes K, Saltzherr TP, Luitse JSK, et al. Indications for total-body computed tomography in blunt trauma patients: a systematic review. Eur J Trauma Emerg Surg 2017; 43: 35-42.

3. Wurmb TE, Frühwald P, Hopfner W, et al. Whole-body multislice computed tomography as the primary and sole diagnostic tool in patients with blunt trauma: searching for its appropriate indication. Am J Emerg Med 2007; 25: 1057-1062.

4. Babaud J, Ridereau-Zins C, Bouhours G, et al. Benefit of the Vittel criteria to determine the need for whole body scanning in a severe trauma patient. Diagn Interv Imaging 2012; 93: 371-379.

5. Ching-Hua H, Yi-Chun C, Shiun-Yuan H, et al. Defining polytrauma by abbreviated injury scale $\geq 3$ for at least two body regions is insufficient interms of short-term outcome: A cross-sectional study at a level I trauma center. Biomed J 2018; 41: 321-327.

6. Smith CM, Woolrich-Burt L, Wellings R, et al. Major trauma CT scanning: the experience of a regional trauma centre in the UK. Emerg Med J 2011; 28: 378-382.

7. Adiotomre A, Chopra A, Kirwadi A, et al. Results from the first year as a major trauma radiology unit in the UK. Clin Radiol 2014; 69: 812-821.

8. Gupta M, Schriger DL, Hiatt JR, et al. Selective use of computed tomography compared with routine whole body imaging in patients with blunt trauma. Ann Emerg Med 2011; 58: 407-416.

9. Bardon M, Young N, Sindhusake P, et al. Correlation of CT findings remote from prime area of interest: a multitrauma study. Open Access Emerg Med 2012; 4: 75-83.
10. Salim A, Sangthong B, Martin M, et al. Whole body imaging in blunt multisystem trauma patients without obvious signs of injury: results of a prospective study. Arch Surg 2006; 141: 468-473.

11. Huber-Wagner S, Lefering R, Qvick LM, et al. Effect of whole-body CT during trauma resuscitation on survival: a retrospective, multicentre study. Lancet 2009; 373: 1455-1561.

12. Huber-Wagner S, Biberthaler P, Häberle S, et al. Whole-body CT in haemodynamically unstable severely injured patients - a retrospective, multicentre study. PLoS One 2013; 8: e68880.

13. Yeguiayan JM, Yap A, Freysz M, et al. Impact of whole-body computed tomography on mortality and surgical management of severe blunt trauma. Crit Care 2012; 16: R101.

14. Goslings JC, Sierink JC, Treskes K, et al. Immediate total-body CT scanning versus conventional imaging and selective CT scanning in patients with severe trauma (REACT-2): a randomised controlled trial. Lancet 2016; 388: 673-683.

15. Frenzel S, Krenn P, Heinz T, et al. Does the applied polytrauma definition notably influence outcome and patient population? - a retrospective analysis. Scand J Trauma Resusc Emerg Med 2017; 25: 87.

16. Banerjee P, Rudra S, Ghosh M, et al. CT scans in primary survey for polytrauma patients. Adv Comput Tomogr 2013; 2: 46-51.

17. Błaż M, Palczewski P, Gołębiowski M, et al. Is whole-body trauma MDCT justified in patients in good clinical condition but with dangerous trauma mechanism? Pol J Radiol 2009; 74: 16-20.

18. Davies RM, Scrimshire AB, Sweetman L, et al. A decision tool for whole-body CT in major trauma that safely reduces unnecessary scanning and associated radiation risks: An initial exploratory analysis. Injury 2015; 47: 43-49. 